Supporting Information

\title{
Simultaneous Sensing of Force and Current Signals to Recognize Proteinogenic Amino Acids in a Single-molecule Level
}

Changxiong Huang ${ }^{1}$, Xiaohong $Z \mathrm{Ch}^{1}$, Na $\mathrm{Li}^{1}$, Xinyao $\mathrm{Ma}^{1}$, Zhen $\mathrm{Li}^{2}$ *, Jun Fan ${ }^{1,3}$ *

${ }^{1}$ Department of Materials Science and Engineering, City University of Hong Kong, Kowloon 999077, Hong Kong, China

${ }^{2}$ School of Materials Science and Engineering, China University of Petroleum (East China), Qingdao, Shandong 266580, China

3 Center for Advanced Nuclear Safety and Sustainable Development, City University of Hong Kong, Kowloon 999077, Hong Kong, China

\section{Corresponding Author}

*Z. Li: lizhenew@gmail.com

*J.Fan: junfan@cityu.edu.hk 


\section{S1 Simulation Methods}

Models and Simulation Setup. All MD simulations are performed on GROMACS 5.1.4 simulation platform. ${ }^{1}$ CHARMM36 force field ${ }^{2}$ is used for protein, TIP3P water and ions. And the trajectories are analyzed in VMD software. ${ }^{3}$ In accordance with the published works, ${ }^{4-6}$ the carbon atoms in graphene is treated as aromatic carbons (CA atom type) in CHARMM36. Figure 1 shows the simulation model. A two-layered graphene membrane with interlayer distance $3.4 \AA$ is used in this work for the reasons detailed in Supporting Information (SI) S2. The graphene membrane is constrained at its initial position in the whole simulations. The initial box of all the systems is $6.3 \times 6 \times 16 \mathrm{~nm}^{3}$ with $2 \mathrm{M}$ potassium chloride $(\mathrm{KCl})$ aqueous solution. Periodic boundary condition is applied in $x, y$, and $z$ directions. Particle Mesh Ewald (PME) method is used to describe the electrostatic interactions. The van der Waal cutoff is set $12 \AA$. The temperature is set as $295 \mathrm{~K}$. The timestep is $1 \mathrm{fs}$, and the data are output every $2 \mathrm{ps} .20$ homogeneous peptide chains are generated containing 15 identical residues in each, named as $\mathrm{X}_{15}$, where $\mathrm{X}$ represents the 20 types of protein building blocks, as shown in Figure 3a. The $E_{15}$ and $D_{15}$ chains are negatively charged, while the $R_{15}$ and $K_{15}$ are positively charged. The other 16 chains are neutral. As for the MD simulation protocols, all systems are firstly equilibrated in NVT (constant number of atoms, volume, and temperature) for 10ns and NPT ensemble (constant number of atoms, pressure, and temperature) for $20 \mathrm{~ns}$ following an energy minimization for 30000 steps, where the $\mathrm{C}$ terminus of the protein is constraint $5 \AA$ below the graphene membrane. Then the production simulations are run for $\sim 90$ ns by applying constant-velocity MD in NVT 
ensemble with pulling velocity $1 \AA /$ ns to collect signals, how the pulling velocity is decided is detailed in SI S2. The external electric field is set $60 \mathrm{mV} / \mathrm{nm}$, generating a bias about $\sim 960 \mathrm{mV}$, to induce ionic currents.

As for the simulation of the ion distribution in the systems of charged peptides, all the equilibration processes are the same as the sequencing model except that the production MD is simulated for 30ns in NVT ensemble. The last 20ns are used to analyze the ion distributions in solution. The backbone atoms of the four peptides are restrained at their initial configuration with a force constant $1000 \mathrm{~kJ} \cdot \mathrm{mol}^{-1} \cdot \mathrm{nm}^{-1}$ during the whole simulation.

Data Analysis. The sensed force and current signals are shown in Figure1c to 1e in the main text. The trajectories in the production MD are output every $2 \mathrm{ps}$. The pulling force profile is generated by default during production simulations, and then averaged every 400 ps. As for judging the residue translocation, the translocation occurs when its Center of Mass (COM) is exactly transcending the graphene plane. The translocation behavior shows a stepwise way so that the dwell time of a residue is defined as the time interpolation from one step to the next in the colored area in Figure $1 \mathrm{~b}$. The ionic currents are calculated between every frame using the equation below: ${ }^{7-8}$

$$
I(t)=\frac{\Delta Q}{\Delta t}=\frac{\sum_{i=1}^{N} q_{i}\left[Z_{i}(t+\Delta t)-Z_{i}(t)\right]}{\Delta t \cdot L_{Z}}
$$

where $N$ is the total number of the charged ions, $\Delta t$ is the time interval between every frame (here $2 \mathrm{ps}$ ), $q_{i}$ and $Z_{i}$ are the charge amount and the $z$ coordinate of the ion number 
$i$, respectively. The periodic boundary conditions are also considered during the calculation. Thereafter, the ionic currents are averaged every $400 \mathrm{ps}$ to obtain the ionic current profile. To ensure the same sampling frequency for all residues, the ionic current is further sampled with a $100 \mathrm{MHz}$ frequency $(10 \mathrm{~ns} \text { per point })^{9}$. The data for analysis is shown in the rainbow area in Figure 1 of the main text.

The number of peptide atoms (NA) is also calculated when a peptide translocates through the nanoslit. It is defined as the number of peptide atoms within a graphene nanoslit. ${ }^{10}$ More specifically, the size of the nanoslit is $x \times y \times z=7 \times 26 \times 6.8 \AA^{3}$ so that the NA refers to the atoms within a $6.8 \AA$-thick graphene nanoslit, as shown in Figure $4 c$.

\section{S2 The parameter optimization for simulation system}

Graphene thickness. The thickness of 2D materials sensor can influence the detecting signals. To decide the thickness or layers of graphene, a pre-simulation calculation is made on a selected peptide with 22 residues and 20 types of amino acids, whose sequence from n' terminus (n ter) to c' terminus (c ter) is n'-WWYR FHME KQDN LICT VPSA GG-c'. Figure S1 shows the distances among the adjacent residues in this peptide. In the backbone of this peptide, every residue contributes one alpha carbon atom. The distances among alpha carbons are around $4 \AA$. Besides, the center of mass (COM) distances among adjacent residues range from 4 to $9 \AA$, averaging around $6 \AA$. As for the sensor, a single layer graphene is $3.4 \AA$. To ensure the single-residue resolution of the nanoslit sensor, the thickness should be no more than the doubled 
minimum COM distance, around $8 \AA$. The single-layered (3.4 $\AA$ ) or double-layered (6.8 Å) graphene nanoslit can fulfill this requirement.
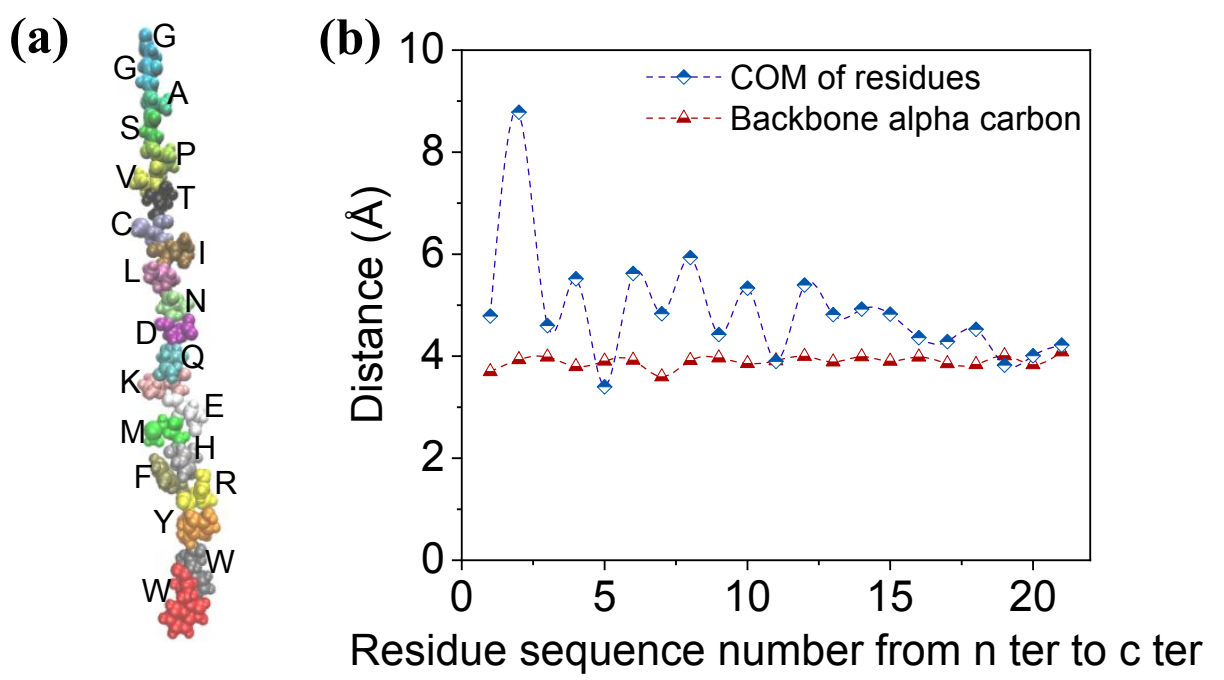

Figure S1 A preliminary estimation of graphene nanoslit thickness by calculating the distances between adjacent residues. (a) The illustration of the selected peptide with 22 residues constituting 20 types of amino acids. The sequence from $\mathrm{n}$ terminus ( $\mathrm{n}$-ter) to c terminus (c-ter) is n'-WWYRFHMEKQDNLICTVPSAGG-c'. (b) The distances among adjacent residues. Both distances, among the center of mass (COM) and the alpha carbon in backbone, were calculated. The sequence numbers in $X$ axis represent the counted residue sequence from n-ter to c-ter.

The double-layered graphene sensor is selected because much work has shown that the thicker sensors have better signal-to-noise ratio, ${ }^{11-12}$ and the DNA nucleotides can be distinguished from both ionic current and dwell time by a double-layered graphene sensor instead of the single-layered. ${ }^{13}$ It is also demonstrated that a single- 
layered graphene is too thin for a DNA to reliably translocate the nanopore because the fracture of the dwell time of a base is too low $(<\sim 36 \%),{ }^{14}$ which will result in a stochastic pore blocking. Based on the simulation works mentioned above, a doublelayered graphene is supposed to be more suitable than the single-layered, meanwhile the thickness of double-layered graphene $(6.8 \AA)$ is also comparable in size to the averaged COM distance among the adjacent residues. As a result, the thickness of the sensor is determined as double-layered graphene.

Pulling velocity. The pulling velocities affect the data sampling when collecting the force and current signals. To get the suitable pulling velocity, four velocities are set, which are $0.2 \AA / \mathrm{ns}, 0.5 \AA / \mathrm{ns}, 1 \AA / \mathrm{ns}$ and $5 \AA / \mathrm{ns}$, in turn. The peptide $\mathrm{A}_{15}$ is set as the model peptide in the constant velocity molecular dynamics (CVMD) simulation and the general simulation setup is the same as detailed in S1 Simulation Methods.

Figure S2 shows the results under different pulling velocities using $A_{15}$ as model peptide. Figure S2a is the pulling force profiles, from which we can see that the pulling force peaks are close to each other, around $150 \mathrm{~kJ} \cdot \mathrm{mol}^{-1} \cdot \mathrm{nm}^{-1}$. Figure S2b is the counted residues translocated through the graphene nanoslit. Under all the four velocities, the residues translocate the nanoslit in a one-by-one and step-by-step behavior. Figure S2c shows the ionic currents with 2 ps per frame and 400 ps averaging. The ionic current curve of $v=5 \AA / n$ s is not shown because its fluctuation range is one magnitude higher than those of the other three. 

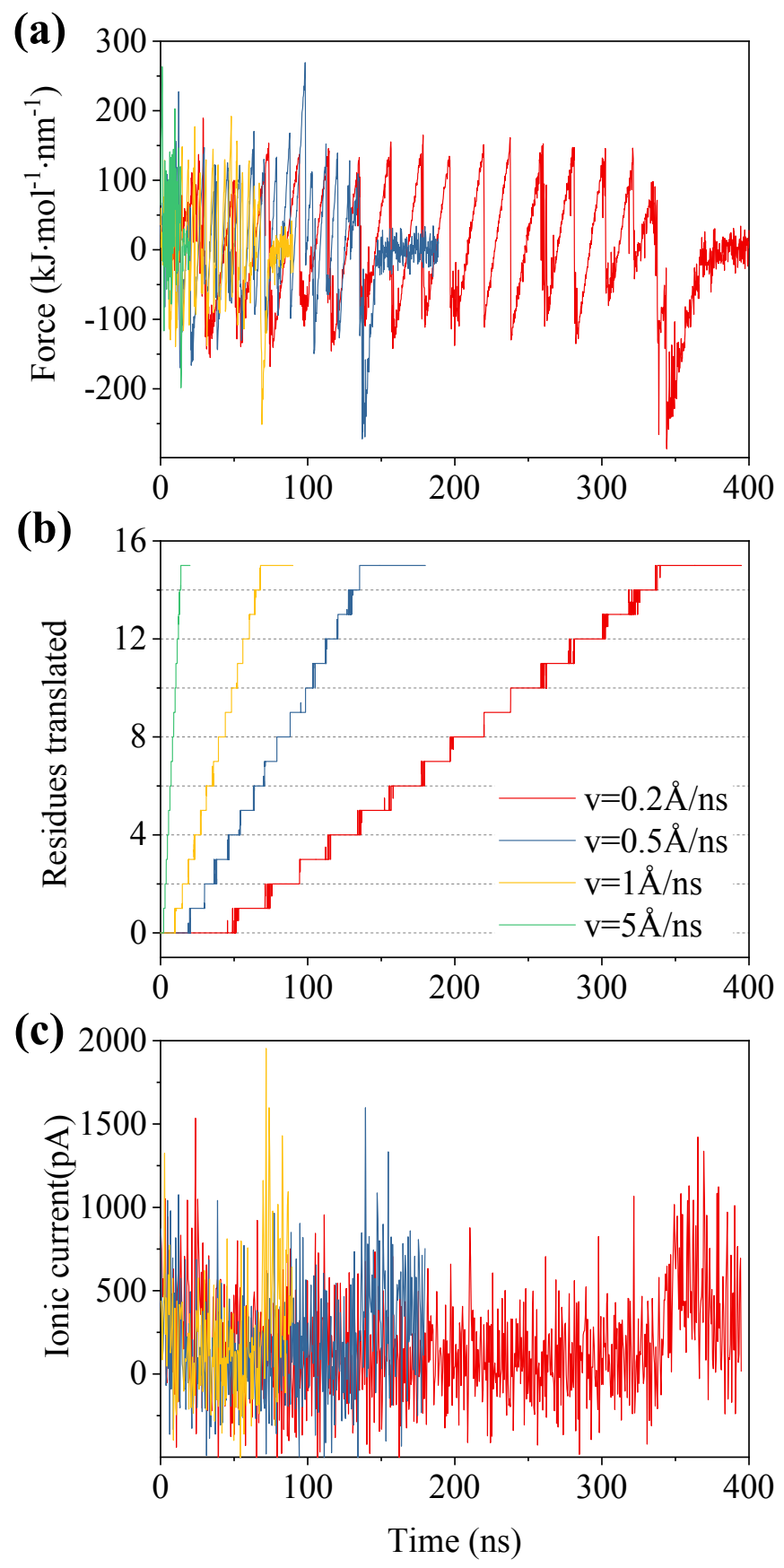

Figure S2 The determination of pulling velocity in CVMD. Four velocities were simulated, which are $0.2 \AA / \mathrm{ns}, 0.5 \AA / \mathrm{ns}, 1 \AA / \mathrm{ns}$ and $5 \AA / \mathrm{ns}$. (a) The force profile, (b) the counted number of translocated residues, and (c) the ionic current profile during CVMD under different pulling velocities. The $\mathrm{A}_{15}$ peptide is the model peptide. 

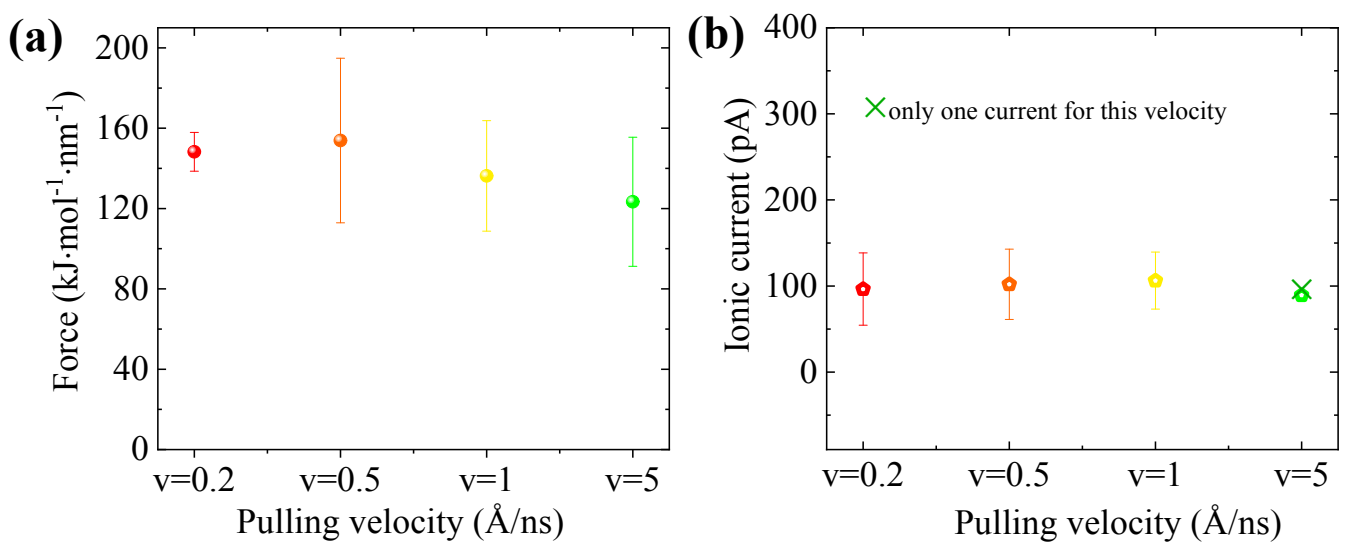

Figure S3 The averaged pulling forces and currents of the $A_{15}$ peptide under different pulling velocities. (a) The pulling forces and (b) the ionic currents. A sampling frequency of $100 \mathrm{MHz}$ was applied to get the ionic currents. There is only one current signal of $v=5 \AA / n$ s under this sampling frequency.

To clearly show the effects of pulling forces on detecting signals, the averaged pulling forces and ionic currents are also calculated with sampling frequency $100 \mathrm{MHz}$ and shown in Figure S3. Figure S3a is the averaged pulling forces, it is clearly seen that the four pulling forces are close to each other, which show that the selected pulling velocities have little effect on detecting pulling forces. Figure $\mathrm{S} 3 \mathrm{~b}$ is the averaged ionic currents. There is only one data for $v=5 \AA / \mathrm{ns}$ because the simulation time is $20 \mathrm{~ns}$ under this velocity and there is not enough time to sample the ionic currents with error bars. As the other three velocities, their ionic currents are in the same range. And the averaged values are close to each other with similar errors. Three pulling velocities fulfills the requirements of data sampling. But if we choose lower pulling velocities, 
more sampling data can be obtained, but the noises or the errors could not be significantly reduced within the selected velocities. Finally, to save computing time and ensure enough sampling data, we adopt $v=1 \AA / n$ s as our pulling velocity in the simulations of sequencing 20 homogeneous peptide chains.

\section{S3 The properties and signals of 20 amino acids/residues}

As supporting information to the Figure 2 in the main text, the detected force and current signals with error bars are shown in Figure S4. The colors in Figure S4 represent the same residues/amino acids in Figure 3. A same grouping can also be clearly seen as in the main text and the similar colored area contains residues with similar properties and signals.

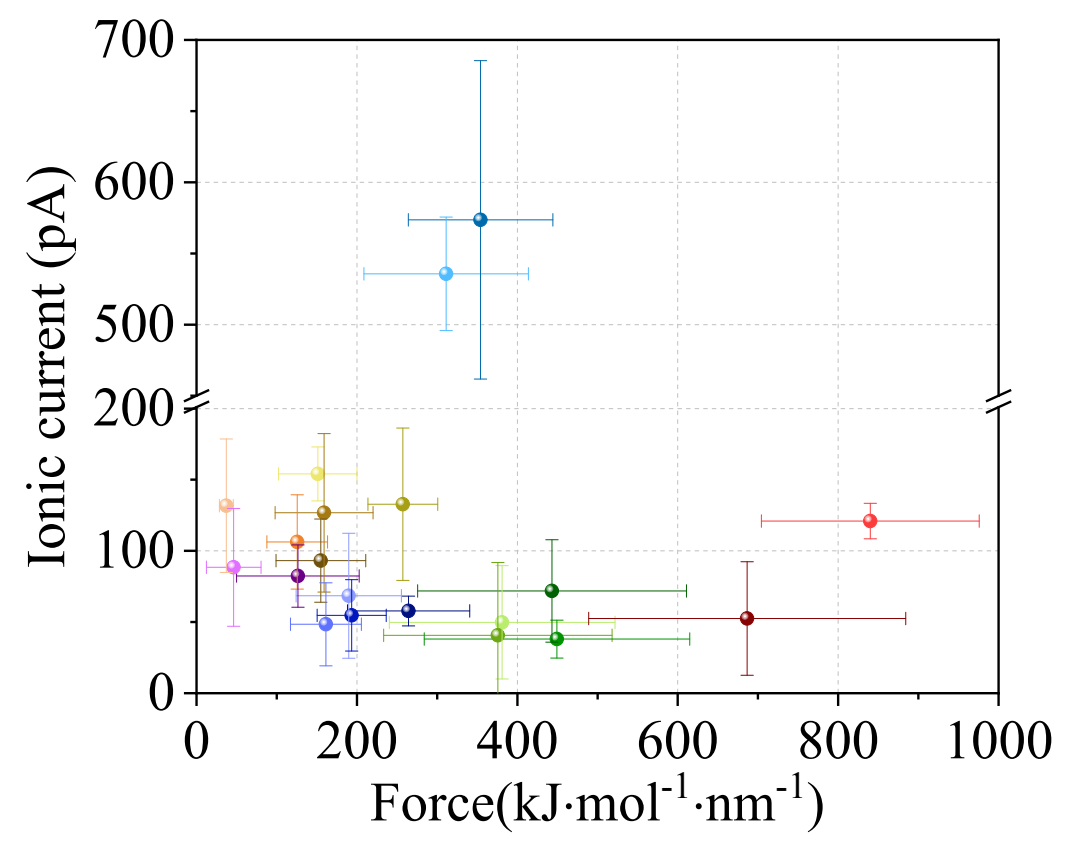

Figure S4 The force and current signals of 20 types amino acids with error bars. The colors represent the same residues or amino acids as Figure 3 in main text. 
Table S1 Properties and signals of the 20 amino acids.

\begin{tabular}{|c|c|c|c|c|c|c|c|c|}
\hline Number & $\begin{array}{l}\text { Amino } \\
\text { acids }\end{array}$ & Synonym & $\begin{array}{l}\text { Mass } \\
(\text { da })^{4} \text { a) }\end{array}$ & $\begin{array}{c}\text { VdW } \\
\text { volume } \\
(\AA 3)^{5} \text { b) }\end{array}$ & Charge & $\begin{array}{l}\text { Molecule } \\
\text { formula }\end{array}$ & $\begin{array}{c}\text { Force } \\
\left(\mathbf{k J} \cdot \mathbf{m o l}^{-}\right. \\
\left.{ }^{1} \cdot \mathbf{n m}^{-1}\right)\end{array}$ & $\begin{array}{c}\text { Ionic } \\
\text { current } \\
\text { (pA) }\end{array}$ \\
\hline 1 & Glycine & GLY, G & 57.052 & 48 & 0 & $\mathrm{C} 2 \mathrm{H} 5 \mathrm{O} 2 \mathrm{~N}$ & $37.07 \pm 8.63$ & $\begin{array}{c}131.7 \pm \\
46.92\end{array}$ \\
\hline 2 & Alanine & ALA, A & 71.0788 & 67 & 0 & $\mathrm{C} 3 \mathrm{H} 7 \mathrm{O} 2 \mathrm{~N}$ & $\begin{array}{c}125.52 \pm \\
37.85\end{array}$ & $\begin{array}{c}106.29 \pm \\
33.14\end{array}$ \\
\hline 3 & Serine & SER, S & 87.0782 & 73 & 0 & $\mathrm{C} 3 \mathrm{H} 7 \mathrm{O} 3 \mathrm{~N}$ & $\begin{array}{c}151.22 \pm \\
48.85\end{array}$ & $\begin{array}{c}154.07 \pm \\
18.93\end{array}$ \\
\hline 4 & Proline & PRO, P & 97.1167 & 90 & 0 & $\mathrm{C} 5 \mathrm{H} 9 \mathrm{O} 2 \mathrm{~N}$ & $\begin{array}{c}840.06 \pm \\
135.71\end{array}$ & $\begin{array}{c}120.94 \pm \\
12.51\end{array}$ \\
\hline 5 & Valine & VAL, V & 99.1326 & 105 & 0 & $\mathrm{C} 5 \mathrm{H} 11 \mathrm{O} 2 \mathrm{~N}$ & $\begin{array}{c}381.06 \pm \\
140.76\end{array}$ & $\begin{array}{c}49.8 \pm \\
39.81\end{array}$ \\
\hline 6 & Threonine & THR, T & $\begin{array}{c}101.105 \\
1\end{array}$ & 93 & 0 & $\mathrm{C} 4 \mathrm{H} 9 \mathrm{O} 3 \mathrm{~N}$ & $\begin{array}{c}257.29 \pm \\
43.50\end{array}$ & $\begin{array}{c}132.8 \pm \\
53.56\end{array}$ \\
\hline 7 & Cysteine & CYS, C & $\begin{array}{c}103.144 \\
8\end{array}$ & 86 & 0 & $\mathrm{C} 3 \mathrm{H} 7 \mathrm{O} 2 \mathrm{NS}$ & $\begin{array}{c}155.02 \pm \\
55.95\end{array}$ & $\begin{array}{c}93.12 \pm \\
29.25\end{array}$ \\
\hline 8 & Isoleusine & ILE, I & $\begin{array}{c}113.159 \\
5\end{array}$ & 124 & 0 & $\mathrm{C} 6 \mathrm{H} 13 \mathrm{O} 2 \mathrm{~N}$ & $\begin{array}{c}686.54 \pm \\
197.67\end{array}$ & $\begin{array}{c}52.48 \pm \\
39.84\end{array}$ \\
\hline 9 & Leucine & LEU, L & $\begin{array}{c}113.159 \\
5\end{array}$ & 124 & 0 & $\mathrm{C} 6 \mathrm{H} 13 \mathrm{O} 2 \mathrm{~N}$ & $\begin{array}{c}189.80 \pm \\
65.68\end{array}$ & $\begin{array}{c}68.45 \pm \\
43.93\end{array}$ \\
\hline 10 & Aspragine & ASN, N & $\begin{array}{c}114.103 \\
9\end{array}$ & 96 & 0 & $\mathrm{C} 4 \mathrm{H} 8 \mathrm{O} 3 \mathrm{~N}$ & $\begin{array}{c}158.95 \pm \\
61.07\end{array}$ & $\begin{array}{c}126.77 \pm \\
55.69\end{array}$ \\
\hline 11 & Aspartic & ASP, D & $\begin{array}{c}115.088 \\
6\end{array}$ & 91 & $-e$ & $\mathrm{C} 4 \mathrm{H} 7 \mathrm{O} 4 \mathrm{~N}$ & $\begin{array}{c}311.24 \pm \\
102.59\end{array}$ & $\begin{array}{c}535.63 \pm \\
39.9\end{array}$ \\
\hline 12 & Glutamine & GLN, Q & $\begin{array}{c}128.130 \\
8\end{array}$ & 114 & 0 & $\mathrm{C} 5 \mathrm{H} 10 \mathrm{O} 3 \mathrm{~N} 2$ & $\begin{array}{c}193.52 \pm \\
43.09\end{array}$ & $\begin{array}{c}54.71 \pm \\
25.05\end{array}$ \\
\hline 13 & Lysine & LYS, K & $\begin{array}{c}128.174 \\
2\end{array}$ & 135 & $+\mathrm{e}$ & $\mathrm{C} 6 \mathrm{H} 14 \mathrm{O} 2 \mathrm{~N} 2$ & $\begin{array}{c}46.35 \pm \\
34.10\end{array}$ & $\begin{array}{c}88.42 \pm \\
41.38\end{array}$ \\
\hline 14 & Glutamic & GLU, E & $\begin{array}{c}129.115 \\
5\end{array}$ & 109 & $-\mathrm{e}$ & $\mathrm{C} 5 \mathrm{H} 9 \mathrm{O} 4 \mathrm{~N}$ & $\begin{array}{c}354.16 \pm \\
90.12\end{array}$ & $\begin{array}{c}573.55 \pm \\
111.84\end{array}$ \\
\hline 15 & $\begin{array}{c}\text { Methionin } \\
\mathrm{e}\end{array}$ & MET, M & $\begin{array}{c}131.198 \\
6\end{array}$ & 124 & 0 & C5H11O2NS & $\begin{array}{c}161.30 \pm \\
44.15\end{array}$ & $\begin{array}{c}48.43 \pm \\
29.17\end{array}$ \\
\hline 16 & Histidine & HIS, H & $\begin{array}{c}137.141 \\
2\end{array}$ & 118 & 0 & $\mathrm{C} 6 \mathrm{H} 9 \mathrm{O} 2 \mathrm{~N} 3$ & $\begin{array}{c}264.31 \pm \\
76.21\end{array}$ & $\begin{array}{r}57.8 \pm \\
10.45\end{array}$ \\
\hline 17 & $\begin{array}{c}\text { Phenylalan } \\
\text { ine }\end{array}$ & PHE, F & $\begin{array}{c}147.176 \\
6\end{array}$ & 135 & 0 & $\mathrm{C} 9 \mathrm{H} 11 \mathrm{O} 2 \mathrm{~N}$ & $\begin{array}{c}375.81 \pm \\
142.39\end{array}$ & $\begin{array}{c}40.62 \pm \\
51.17\end{array}$ \\
\hline 18 & Arginine & ARG, R & $\begin{array}{c}156.187 \\
6\end{array}$ & 148 & $+\mathrm{e}$ & $\mathrm{C} 6 \mathrm{H} 14 \mathrm{O} 2 \mathrm{~N} 4$ & $\begin{array}{c}126.39 \pm \\
76.50\end{array}$ & $\begin{array}{c}82.31 \pm \\
21.95\end{array}$ \\
\hline 19 & Tyrosine & TYR, Y & 163.176 & 141 & 0 & $\mathrm{C} 9 \mathrm{H} 11 \mathrm{O} 3 \mathrm{~N}$ & $\begin{array}{c}449.40 \pm \\
165.57\end{array}$ & $\begin{array}{c}38.02 \pm \\
13.32\end{array}$ \\
\hline 20 & Tryptopha & TRP, W & 186.213 & 163 & 0 & $\mathrm{C} 11 \mathrm{H} 12 \mathrm{~N} 2 \mathrm{O} 2$ & $443.27 \pm$ & $71.85 \pm$ \\
\hline
\end{tabular}




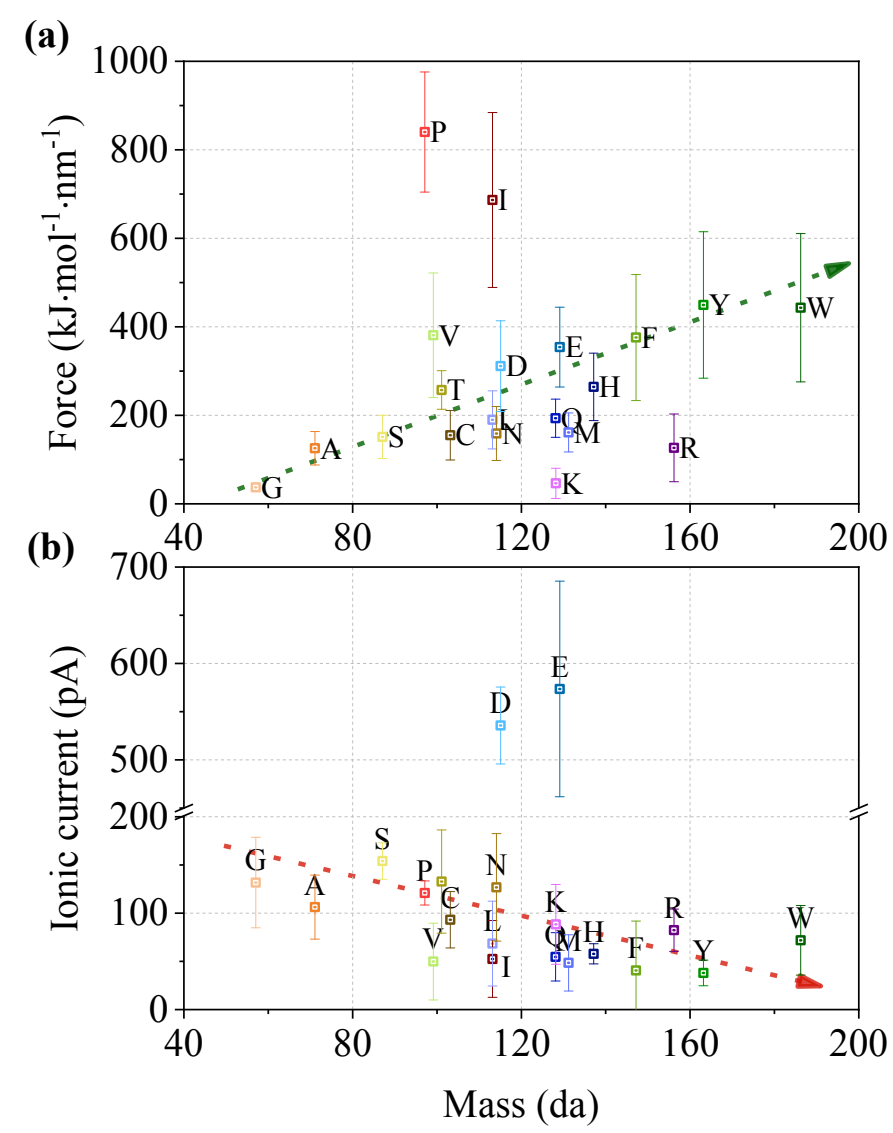

Figure S5 The relationship between the properties and the signals of the 20 amino acids.

(a)The molecular mass versus the forces, (b) the molecular mass ${ }^{15}$ versus the currents. A general pattern is that the heavier amino acids have lower ionic currents and higher pulling forces.

Apart from the figure showing the grouping of 20 amino acids, the properties and the detecting signals are also summarized. Table $\mathrm{S} 1$ shows the names, abbreviations, atom mass, ${ }^{15}$ van der Waals (vdW) volumes, ${ }^{16}$ charges in solution, ${ }^{17}$ molecule formula, and their corresponding force and current signals with errors detected by the graphene 
nanoslit sensor. There are only four charged residues: $\mathrm{K}$ and $\mathrm{R}$ residues are positively charged, D and E are negatively charged. The other 16 residues are uncharged in this simulation work, although some may have several protonation states.

Figure S5, together with Figure 3c and 3d, show the relationships among properties and single detecting signals of 20 amino acids. From Figure S5a, the pulling forces of the group containing $\mathrm{P}$ and $\mathrm{I}$ is higher than the others while the positively charged $\mathrm{K}$ and $\mathrm{R}$ residues are lower. As for the rest residues, a trend that the heavier residues have higher pulling forces is observed. From Figure S5b, the ionic currents of negatively charged $\mathrm{D}$ and $\mathrm{E}$ are higher than the others. As for the remaining residues, the trend is that heavier residues have lower ionic currents. Together with Figure 3 of the vdW volumes versus force and current signals, a general pattern that the heavier and larger residues have higher pulling forces and lower ionic currents can be observed.

\section{S4 The analysis of anomaly signals.}

When detecting the force and current signals of the amino acids, some exceptions beyond the general patterns occur. Their underlying causes are probed and detailed in the main text, and some complementary data are shown in this part.

Figure S6 shows the pulling force profile and the translocation process of peptide $\mathrm{I}_{15}$ through $X 7 Y 26$ nanoslit. From Figure S6a, there are several force peaks obviously larger than the other peaks, which corresponds well with the translocation events in Figure S6b, where two residues translocate through the graphene nanoslit in one 
translocation event. This behavior is the same as that of peptide $\mathrm{P}_{15}$, resulting in the extremely high pulling forces.

(a)

(b)
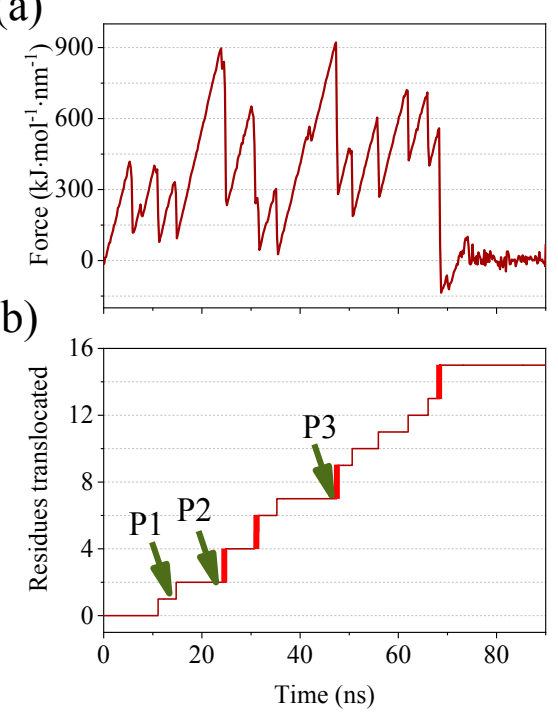

(c)

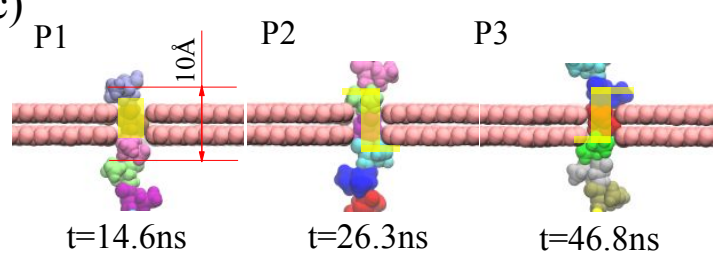

(d)

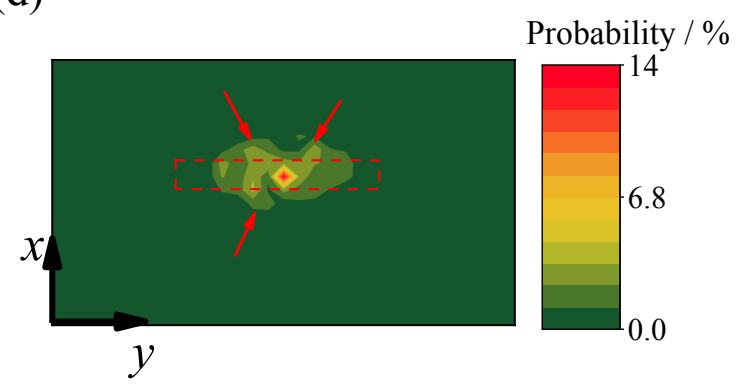

Figure S6 (a) The pulling forces and (b) the counted residues of peptide $\mathrm{I}_{15}$ translocated through the $X 7 Y 26$ nanoslit during CVMD. There are two residues translocating through graphene nanoslit in one single translocation event, which are bolded in red. The three points, P1, P2, and P3 label the translocation snapshots in (c), where there are three conformations showing how the residues translocate through nanoslit, where a sandwich structure caused by the side chain is formed, resulting in high pulling force. (d) The normalized density or probability of protein in the vicinity of graphene nanoslit. The detected area is in the range $15 \AA<x<45 \AA, 15 \AA<y<55 \AA$, and $z$ within $5 \AA$ of graphene plane. The protein atoms also show up out of the nanoslit-confined space, meaning that some side chains absorb on the graphene plane and form a sandwich structure. 
Further investigation on the translocation process is performed to show how the high pulling forces of I residues come. There are three points labelled in Figure S6b. $\mathrm{P} 1$ is the point before a single residue translocates through nanoslit, while P2 and P3 are the points where two residues as a whole translocate through the nanoslit. The snapshots of the three points are shown in Figure S6c. At P1, a single residue is in nanoslit and prepares itself for the translocation through nanoslit. At P2, the purple residue is in the nanoslit, but the side chains of its former and latter residues adsorb on the graphene plane and form a sandwiched structure. This phenomenon can also be observed at P3, whose sandwiched structure is on the opposite direction, as labelled by the yellow zone (Figure S6c). To confirm this explanation, the normalized density distribution (probability in Figure S6d) of the heteropeptide is calculated during the translocation process. The selected time is from $10 \mathrm{~ns}$ to $50 \mathrm{~ns}$, and the area of the calculation is in the range $15 \AA<x<45 \AA, 15 \AA<y<55 \AA$, and $z$ within $5 \AA$ of graphene plane. In Figure S6d, most residue atoms are within the confined space of graphene nanoslit during translocation. But there are still some atoms out of the confined space, as labelled by the red arrows. Taken together S6c and S6d, these areas are caused by the adsorption of side chains on graphene plane, and the areas represent the locations of sandwich structures. It seems that the sandwich structures stochastically exist during the translocation process. How much effect this structure may have on signals when sequencing different proteins needs deeper probes.

Figure $\mathrm{S} 7$ shows the ion distribution in the vicinity of peptide $\mathrm{E}_{15}$ and $\mathrm{K}_{15}$ peptides in $\mathrm{KCl}$ aqueous solutions. Figure $\mathrm{S} 7 \mathrm{a}$ shows the ion distribution in the $x y$ plane normal 
to the protein axis, and $\mathrm{S} 7 \mathrm{~b}$ is $C(r) / C_{0}$ of cations and anions in the vicinity of charged peptides. The concentration of cations $\left(\mathrm{K}^{+}\right)$near the negatively charged $\mathrm{E}_{15}$ peptide is higher than that of anions $\left(\mathrm{Cl}^{-}\right)$near the positively charged $\mathrm{K}_{15}$ peptide. Meanwhile, the $\mathrm{Cl}^{-}$concentration near $\mathrm{E}_{15}$ is also higher than that of $\mathrm{K}^{+}$concentration near $\mathrm{K}_{15}$. Taken together, the negatively charged peptides adsorb more ions in their vicinities than that of positively charged peptides. As detailed in the main text, this unproportionate adsorbent ability leads to the high concentration of ions in the vicinities of negatively charged peptides, resulting in their exceptionally high ionic currents.
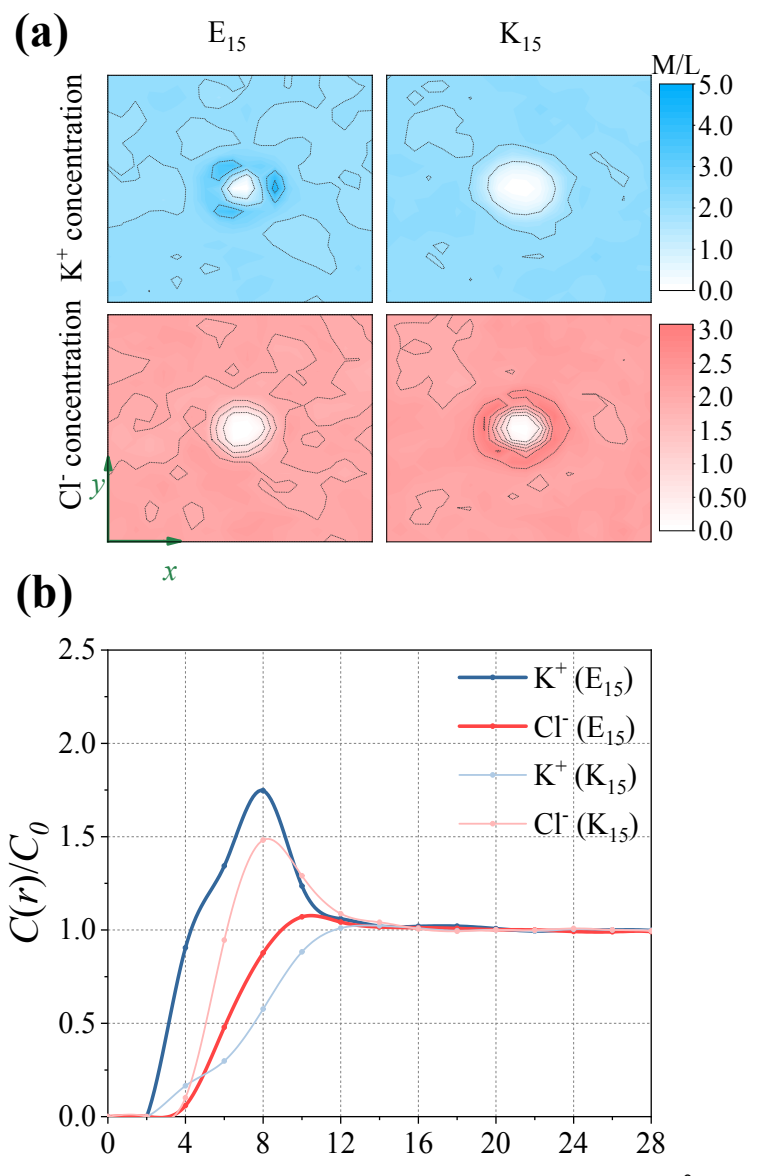

Distance $r$ from the peptide backbone $(\AA)$

Figure S7 Ion distributions in the vicinity of positively charged peptide $\mathrm{K}_{15}$ and negatively charged peptide $\mathrm{E}_{15}$. 


\section{S5 The signals of a heterogeneous peptide}

The pulling force and ionic current signals are collected based on sequencing 20 homogeneous peptides, which shows improved distinguishability to identify different types of amino acids. Whether this approach can work well for identifying the sequence of a heteropolymer is still unknown. To provide further evidence on the validity of simultaneous sensing force and ionic current signals, a heterogeneous peptide with sequence c'-DDDDAAAAKKKKWWWW-n' (D4A4K4W4) is also sequenced based on the afore-mentioned simulation set up. The simulation model and results are shown in Figure S8.

The simulation model of sequencing heterogeneous peptide is shown in Figure S8a. The peptide D4A4K4W4 is pulled through nanoslit under applied electric field and applied force. The pulling forces and ionic current signals are collected and then averaged by their residue types, as shown in Figure S8b. The collected data during the translocation process are shown in Figure S8c to S8e, which in turn are the pulling force profile, the counted number of translocated residues, and the ionic current profile. The colored areas represent the translocation period of different residue types. The residues in the heteropeptide translocate through the nanoslit step by step (Figure S8d). From the force signal (Figure S8c), the force peaks of different residue types are different. From the ionic current curve (Figure S8d), the ionic currents of uncharged A and W residues are significantly lower than those of the charged D and $\mathrm{K}$ residue. Doing averaging to the force peaks and ionic currents of different residue type, a plot is got showing the force-current relationship of different residues in heterogenous peptide 
(Figure $\mathrm{S} 8 \mathrm{~b}$ ). The four residues, $\mathrm{D}, \mathrm{A}, \mathrm{K}$, and $\mathrm{W}$ can be significantly identified in the heterogenous state.

(a)

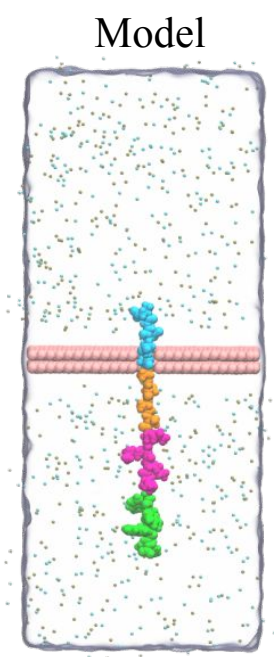

(b)
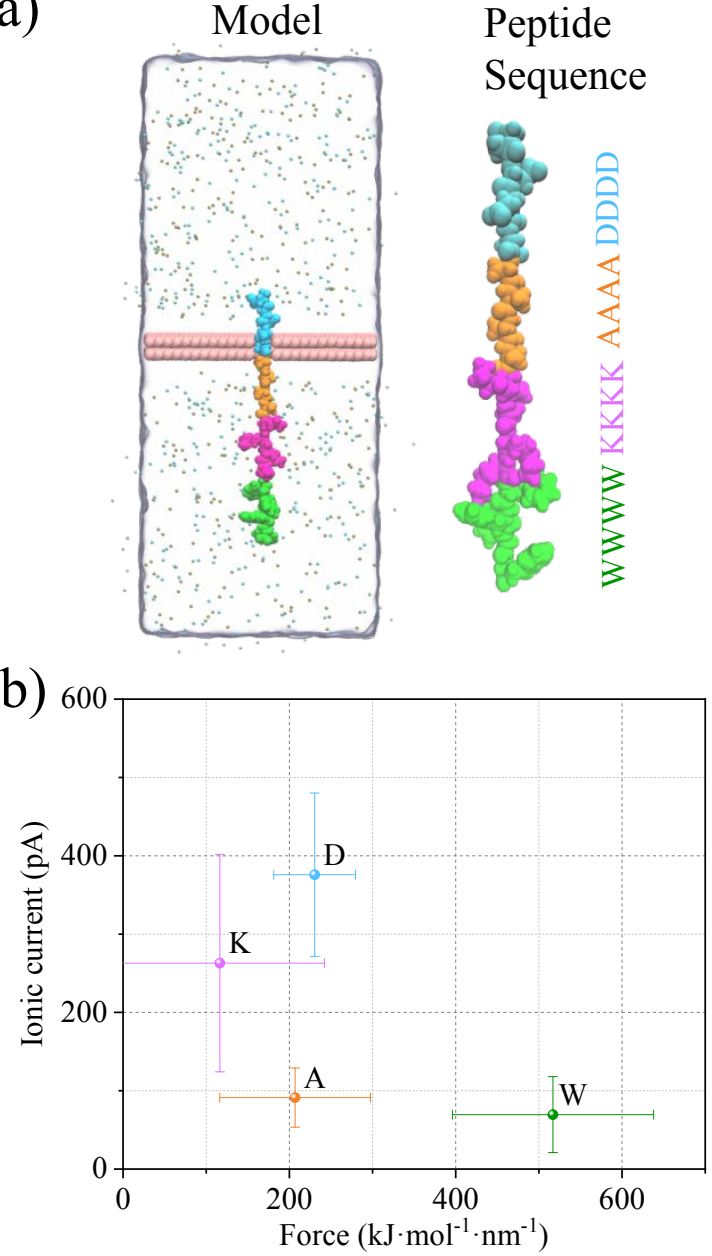

(c)

(d)
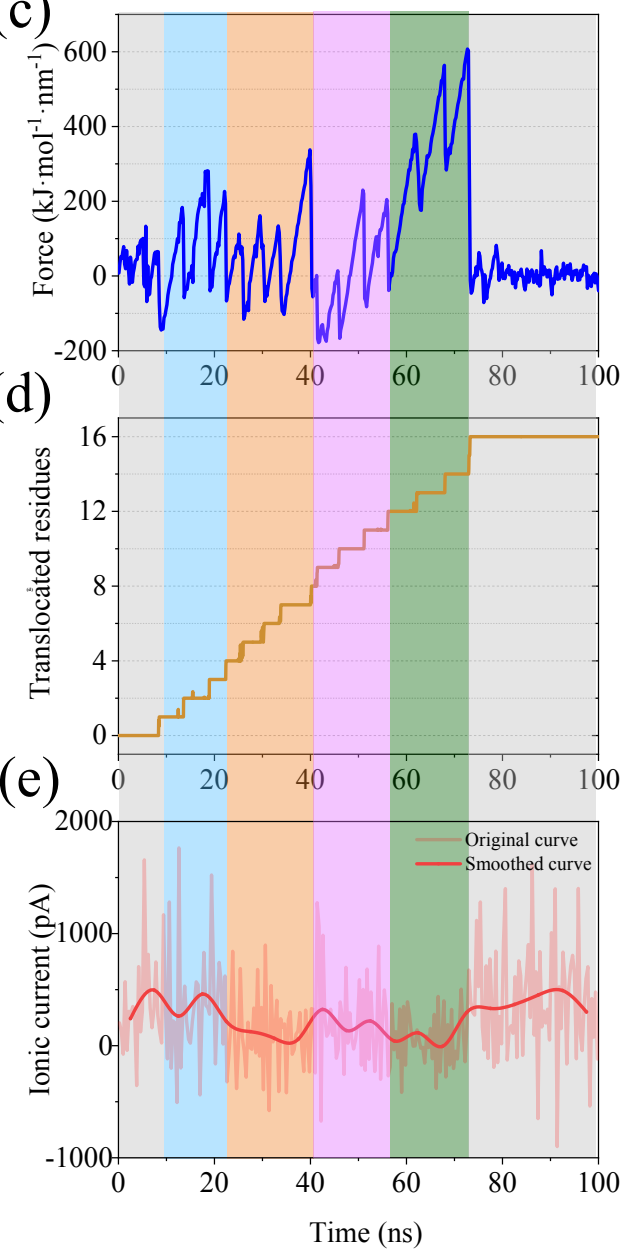

Figure S8 The simulation model and detected signals of sequencing heterogeneous peptide D4A4K4W4. (a) The simulation model showing the process of peptide D4A4K4W4 translocating through graphene nanoslit under applied electric field and pulling force, where different colors represent different types of amino acids. (b) The averaged pulling force peaks and averaged ionic currents of the four amino acids, D, A, $\mathrm{K}$, and W. (c) The pulling force profile, (e) the counted number of translocated residues, and (d) the ionic current profile during the simulation. 
The simulation on applying force and current signals to detect different residue types is performed, results show that four residue types can be identified in the heterogenous state. However, this simulation on heterogenous peptide is preliminary, and further investigation on sequencing the heteropolymers is needed. 


\section{REFERENCES}

(1) Abraham, M. J.; Murtola, T.; Schulz, R.; Páll, S.; Smith, J. C.; Hess, B.; Lindahl, E. GROMACS: High Performance Molecular Simulations through Multi-Level Parallelism from Laptops to Supercomputers. SoftwareX 2015, 1$2,19-25$

(2) Huang, J.; Rauscher, S.; Nawrocki, G.; Ran, T.; Feig, M.; de Groot, B. L.; Grubmuller, H.; MacKerell, A. D., Jr. CHARMM36m: An Improved Force Field for Folded and Intrinsically Disordered Proteins. Nat. methods 2017, 14 (1), 71-73.

(3) Humphrey, W.; Dalke, A.; Schulten, K. VMD: Visual Molecular Dynamics. J. Mol. Graphics 1996, 14 (1), 33-8, 27-8.

(4) Qiu, H.; Sarathy, A.; Leburton, J. P.; Schulten, K. Intrinsic Stepwise Translocation of Stretched ssDNA in Graphene Nanopores. Nano Lett. 2015, $15(12), 8322-30$

(5) Zhang, Z.; Shen, J.; Wang, H.; Wang, Q.; Zhang, J.; Liang, L.; Agren, H.; Tu, Y. Effects of Graphene Nanopore Geometry on DNA Sequencing. J. Phys. Chem. Lett. 2014, 5 (9), 1602-7.

(6) Si, W.; Zhang, Y.; Wu, G.; Kan, Y.; Zhang, Y.; Sha, J.; Chen, Y. Discrimination of Protein Amino Acid or Its Protonated State at SingleResidue Resolution by Graphene Nanopores. Small 2019, 15 (14), e1900036.

(7) Si, W.; Aksimentiev, A. Nanopore Sensing of Protein Folding. ACS Nano 2017, 11 (7), 7091-7100.

(8) Tyagi, A.; Chu, K.; Hossain, M. D.; Abidi, I. H.; Lin, W.; Yan, Y.; Zhang, K.; Luo, Z. Revealing the Mechanism of DNA Passing through Graphene and Boron Nitride Nanopores. Nanoscale 2019, 11 (48), 23438-23448. 
(9) Farshad, M.; Rasaiah, J. C. Molecular Dynamics Simulation Study of Transverse and Longitudinal Ionic Currents in Solid-State Nanopore DNA Sequencing. ACS Appl. Nano Mater. 2020, 3 (2), 1438-1447.

(10)Qiu, H.; Sarathy, A.; Schulten, K.; Leburton, J. P. Detection and Mapping of DNA Methylation with 2D Material Nanopores. npj 2D Mater. Appl. 2017, 1.

(11) Wilson, J.; Sloman, L.; He, Z.; Aksimentiev, A. Graphene Nanopores for Protein Sequencing. Adv. Funct. Mater. 2016, 26 (27), 4830-4838.

(12) Lv, W.; Chen, M.; Wu, R. a. The Impact of the Number of Layers of A Graphene Nanopore on DNA Translocation. Soft Matter. 2013, 9 (3), 960966.

(13) Li, J.; Wang, H.; Li, Y.; Han, K. The Impact of The Number of Layers of the Graphene Nanopores and the Electrical Field On ssDNA Translocation. Mol. Simul. 2017, 43 (4), 320-325.

(14) Wells, D. B.; Belkin, M.; Comer, J.; Aksimentiev, A. Assessing Graphene Nanopores for Sequencing DNA. Nano Lett. 2012, 12 (8), 4117-23.

(15)Knapp, D. R. Mass Spectrometry in the Biological Sciences A. L. Burlingame and S. A. Carr, Editors. J. Am. Soc. Mass Spectrom. 1996, 7 (7), 692-692.

(16) Moradas Ferreira, P. Protein structure: In focus. Biochem. Educ. 1995, 23 (1), 46-46.

(17)Barati Farimani, A.; Heiranian, M.; Aluru, N. R. Identification of Amino Acids with Sensitive Nanoporous MoS2: Towards Machine Learning-Based Prediction. npj 2D Mater. Appl. 2018, 2 (1). 